Probability, Networks and Algorithms

Probability, Networks and Algorithms

Extrapolating and interpolating spatial patterns

M.N.M. van Lieshout, A.J. Baddeley

Report PNA-R01 17 October 31, 2001 
CWI is the National Research Institute for Mathematics and Computer Science. It is sponsored by the Netherlands Organization for Scientific Research (NWO).

CWI is a founding member of ERCIM, the European Research Consortium for Informatics and Mathematics.

CWI's research has a theme-oriented structure and is grouped into four clusters. Listed below are the names of the clusters and in parentheses their acronyms.

\section{Probability, Networks and Algorithms (PNA)}

Software Engineering (SEN)

Modelling, Analysis and Simulation (MAS)

Information Systems (INS)

Copyright (C) 2001, Stichting Centrum voor Wiskunde en Informatica

P.O. Box 94079, 1090 GB Amsterdam (NL)

Kruislaan 413, 1098 SJ Amsterdam (NL)

Telephone +31205929333

Telefax +31205924199

ISSN 1386-3711 


\title{
Extrapolating and Interpolating Spatial Patterns
}

\author{
M.N.M. van Lieshout ${ }^{1}$ and A.J. Baddeley ${ }^{2}$ \\ ${ }^{1} C W I$ \\ P.O. Box 94079, 1090 GB Amsterdam, The Netherlands \\ 2 Department of Mathematics 8 Statistics, \\ University of Western Australia \\ Nedlands WA 6009, Australia
}

\begin{abstract}
We discuss issues arising when a spatial pattern is observed within some bounded region of space, and one wishes to predict the process outside of this region (extrapolation) as well as to perform inference on features of the pattern that cannot be observed (interpolation). We focus on spatial cluster analysis. Here the interpolation arises from the fact that the centres of clustering are not observed. We take a Bayesian approach with a repulsive Markov prior, derive the posterior distribution of the complete data, i.e. cluster centres with associated offspring marks, and propose an adaptive coupling from the past algorithm to sample from this posterior. The approach is illustrated by means of the redwood data set (Ripley, 1977).

2000 Mathematics Subject Classification: 62H11, 62H30, 60D05.

Keywords and Phrases: exact simulation, Cox cluster process, germ-grain model, independent cluster process, Neyman-Scott process, spatial birth-and-death process, Markov point process, missing data.

Note: Work carried out under CWI project PNA4.3 'Stochastic Geometry', ARC large grant A69941083 'Extrapolating and Interpolating Spatial Patterns', and NWO grant CIMS 613.003.100.
\end{abstract}

\section{INTRODUCTION}

Observations of a spatial pattern are typically confined to a bounded region of space, while the original pattern of interest can often be imagined to extend outside. Much attention has been paid to statistical inference for models of the pattern given only the partial observations in the sampling window. Less attention has been given to prediction or extrapolation of the process (i.e. of the same realisation of the process) beyond the window of observation, conditional on the partially observed realisation.

A motivating example is the charting of geological faults encountered during coal mining $[5,16]$. It is of interest to predict the likely presence of geological faults outside the region mined so far, and thereby to choose between various mining strategies. Other examples may be found in image processing, for instance the problem of replicating a texture beyond the region where it has been observed [22] as in the editing of a video image so that a foreground object is removed and replaced seamlessly by the background texture.

Partial observation of a spatial pattern may also include effects such as aggregation by administrative regions, deletion of part of the pattern, and the unobservability of a related pattern. Recovery of full information in this context might be called interpolation; it resem- 
bles a missing data problem. In the mining problem discussed above, mapped charts represent only those parts of geological faults which were physically encountered. Gaps may arise because the mined region is not convex both at its outer boundary and within this boundary, because pillars of unmined material remain. Hence it is of interest to join observed line segments together and to interpret them as part of the same continuous fault zone, a process that is known as 'interpretation' by geologists. As another example, geostatistics deals with predicting values of a spatial random process (e.g. precipitation or pollution measurements) from observations at known locations (see e.g. [18, pp. 207-209, 356-379], [41, 81], [40, 42]), and interpolation techniques have been developed under the name of conditional simulation for Gaussian [40] and other second-order random fields [41, pp. $494 \mathrm{ff}$.], [42], as well as for discrete Markov random field models [11].

Relatively few conditional simulation techniques have been developed for spatial processes of geometric features such as points, line segments and filled shapes. Those that exist are based largely on Poisson processes and the associated Boolean models [47, 49, 57, 86]. A major obstacle is the scarcity of spatial models that are both realistic and tractable for simulation. Some exceptions are the following. There has been much interest in the conditional simulation of oil-bearing reservoirs given data obtained from one or more exploration wells $[15,33]$. The wells are essentially linear transects of the spatial pattern of reservoir sand bodies. Typically the sand bodies are idealised as rectangles with horizontal and vertical sides of independent random lengths, placed at random locations following a Poisson point process. For line segment processes, [16] presents some stochastic models with particular application to modelling geological faults (based largely on Poisson processes), geostatistical inference, and possibilities for conditional simulation; [36] describe a pairwise interaction point process model for swarms of small faults in a fault zone, and $[82,83,56]$ study a line segment process for extracting linear networks from remote sensing images. Some of these authors have correctly noted the sampling bias effect attendant on observing a spatial pattern of geometric features within a bounded window (analogous to the 'bus paradox'). Techniques from stochastic geometry need to be enlisted to check the validity of simulation algorithms.

Extrapolation or interpolation of a spatial pattern entails fitting a stochastic model to the observed data, and computing properties of the conditional distribution of this model given the observed data. We will discuss a variety of stochastic models for patterns of geometric objects, and treat typical issues such as edge effects, occlusion and prediction in some generality. Subsequently, we shall focus on the problem of identifying clusters in a spatial point pattern, which can be regarded as interpolation of a two-type point pattern from observations of points of one type only, the points of the other type being the cluster centres $[6,51,52,54]$. Applications may be found in epidemiology, forestry, archaeology, coal mining, animal territory research, and the removal of land mines.

\section{Formulation AND NOTATION}

In this section we describe the general framework considered throughout. The spatial pattern is a random closed set $[63,84] U$ in $\mathbb{R}^{d}$, typically $d=2$ or 3 . The distribution of $U$ is governed by a parameter $\theta$ in some space $\Theta$. 


\subsection{Germ-grain models}

All models considered in this paper are germ-grain models [84, p. $186 \mathrm{ff}$.] constructed as follows. There is an underlying process $X=\left\{X_{i}, i=1,2, \ldots\right\}$ of germs in $\mathbb{R}^{d}$, each associated with a random compact set $Z_{i}$ (the 'grain') in $\mathbb{R}^{d}$ specified by a parameter in some space $\mathcal{Z}$. The 'complete data' process $W=\left\{\left(X_{i}, Z_{i}\right)\right\}$ consists of pairs of germs with their associated grains and hence can be seen as a marked point process. The union of the translated grains, $U=\bigcup_{i}\left(X_{i}+Z_{i}\right)$, forms the germ-grain model.

We shall be concerned mostly with spatial cluster processes, which can be formulated as germ-grain models where the $X_{i}$ are the cluster centres, $Z_{i}$ is the cluster of points or objects associated with centre $X_{i}$ translated back to the origin (i.e. $Z_{i}$ is a random finite set of geometric objects), and $U$ is the union pattern. We will sometimes refer to the $X_{i}$ as the parents and to $X_{i}+Z_{i}$ as the daughters or offspring of $X_{i}$. If both the cluster centres and their offspring are points, $\mathcal{Z}$ is the space $\mathcal{N}$ consisting of all finite point patterns in $\mathbb{R}^{d}$. The complete data $W$ then consists of the patterns $X$ and $U$ together with information mapping each member of $U$ to its cluster centre in $X$.

Note that if $X=\left\{X_{i}, i=1,2, \ldots\right\}$ is a homogeneous Poisson point process, and the $Z_{i}$ are i.i.d. the random closed set $U$ is a Boolean model [80, pp. 484-502]. The common distribution of $Z_{i}$ is called the distribution of the typical grain; the germs $X_{i}$ play only an indirect role.

In practice, one observes the intersection $Y=U \cap A$ of $U$ with a compact window $A \subseteq \mathbb{R}^{d}$. Mostly the window $A$ is fixed and known. More generally, one may assume that $A$ is an observable random set and condition on it, effectively implying $A$ should be ancillary for $\theta$ and independent of $U$. The requirement that $A$ be observable excludes, for example, random thinning models [84, pp. 132-136], [18, pp. $689 \mathrm{ff}$.]. These are unidentifiable in the sense that one cannot distinguish between a point process of low intensity and a heavily thinned point process of higher intensity, without imposing further assumptions.

\subsection{Problem statement}

The goal is, given data $\mathbf{y}=U \cap A$, to obtain estimates of the conditional expectations of random variables associated with $U$ or $W$. Note that in the latter case, $W$ will contain grains $Z_{i}^{*}$ such that $X_{i}^{*}+Z_{i}^{*}$ hits the boundary of $A$. Hence, any extrapolation technique will have to extend $Z_{i}^{*}$ as well as locate germ-grain pairs not hitting $A$.

It is important to realise that the individual objects $X_{i}+Z_{i}$ in the germ-grain model are not assumed to be observable separately. They are merely an intermediate stage in the construction of the model for the random set $U$. For example, any object $X_{i}+Z_{i}$ which is completely occluded, i.e. contained in the union of other objects, is not observable and may as well be absent. Consequently our analysis must depend only on the union set $U$ and not on the representation of $U$ as a union of objects $X_{i}+Z_{i}$. In other words, if the data image $\mathbf{y}$ can be represented in two different ways

$$
\mathbf{y}=\bigcup_{i=1}^{n}\left(X_{i}+Z_{i}\right) \cap A=\bigcup_{j=1}^{m}\left(X_{j}^{\prime}+Z_{j}^{\prime}\right) \cap A
$$

then inference based on either representation must yield identical results. This rules out mark-correlation techniques [67].

Specialising to spatial cluster analysis, inference focuses on the conditional expected number of clusters, the conditional mean number of points per cluster and the posterior distri- 
bution of centre locations as well as the strength of evidence for clustering. As for occlusion effects, the whole essence of the problem is that we do not know which data points belong to the same cluster. Below, we adopt a Bayesian strategy and base inference on the posterior distribution of $W$ given $\mathbf{y}$. The parameter vector $\theta$ will be estimated by Monte Carlo maximum likelihood $[29,30]$.

\subsection{Edge effects and sampling bias}

Edge effects and sampling bias are bound to arise when a spatial pattern of unbounded extent is observed in a bounded frame [4]. In this section, we illustrate these problems for partial realisations of a Poisson process of geometric objects. Although the Poisson assumption allows for explicit computations, the essential complexities of the general problem are already present.

Thus, assume that the germ process $X=\left\{X_{i}\right\}$ is a homogeneous Poisson point process in $\mathbb{R}^{d}$ with intensity $\lambda>0$, that the grains $Z_{i}$ are i.i.d. random compact sets, and that $A \subseteq \mathbb{R}^{d}$ is a fixed, compact window. We wish to generate a realisation of $U \cap A$. The approach taken will be to sample those objects which wholly or partly intersect $A$, and to clip the resulting pattern to the window $A$.

First, note that a translated grain $X_{i}+Z_{i}$ intersects $A$ if and only if $X_{i} \in A \oplus \check{Z}_{i}$, where $A \oplus B=\{a+b: a \in A, b \in B\}$ is the Minkowski sum of two sets $A, B \subseteq \mathbb{R}^{d}$ and $\check{A}=$ $\{-a: a \in A\}$ is the reflection of $A$ about the origin [63, 80, 84]. Hence, the germ-grain pairs $\left(X_{i}, Z_{i}\right)$ for which $X_{i}+Z_{i}$ hits $A$ form an inhomogeneous Poisson process whose intensity measure has density $\lambda \mathbf{1}\{x \in A \oplus \check{Z}\}$ with respect to the product of Lebesgue measure and the probability distribution of the grains. Write $|\cdot|$ for $d$-dimensional volume. Then the number of objects intersecting $A$ is Poisson distributed with mean

$$
\lambda \mathbb{E}|A \oplus \check{Z}|
$$

where the expectation is with respect to the distribution of the typical grain $Z$, provided (2.1) is finite. Given $n$ objects are present, they are i.i.d. with density $\mathbf{1}\{x \in A \oplus \check{Z}\} / \mathbb{E}|A \oplus \check{Z}|$. Turning to the marginal grain distribution, it should be noted that the grains $Z_{i}$ corresponding to objects which intersect $A$ are not a random sample from the distribution of the typical grain $Z$. Instead, their distribution is weighted in the sense that $Z_{i}$ are i.i.d. with distribution

$$
\mathbb{P}_{A}(Z \in \cdot)=\frac{\mathbb{E}(1\{Z \in \cdot\}|A \oplus \check{Z}|)}{\mathbb{E}|A \oplus \check{Z}|}
$$

where $\mathbb{E}$ denotes the expectation with respect to the distribution of the typical grain $Z$. Thus the sampling bias favours larger grains: a larger object is more likely than a smaller object to intersect $A$. The sampling bias also depends on the geometry and relative orientations of $A$ and $Z$. For further information see [80, 84].

To simulate $U \cap A$, the properties just described can be used if the function $f(Z)=|A \oplus \check{Z}|$ and the distribution (2.2) can be evaluated analytically. In two dimensions, if $A$ is a disc of radius $r$ and $Z$ is a.s. convex with nonempty interior, then by Steiner's formula [79, p. 220]

$$
|A \oplus \check{Z}|=|Z|+r \operatorname{length}(\partial Z)+\pi r^{2}
$$

a.s., where length $(\partial Z)$ denotes the length of the boundary of $Z$. Hence (2.2) is a mixture of the area-weighted, the length-weighted and the unweighted typical grain distribution. 
Of course, if $Z$ is a cluster of points, it is not convex. However, if the diameter of $Z$ is almost surely bounded by $D$ (say), we can generate centres $X_{i} \in A \oplus B_{D}$ where $B_{D}$ is the disc of diameter $D$, form the associated $Z_{i}$ and clip $X_{i}+Z_{i}$ to $A$. Similarly, one can reduce to the case where $A$ is convex, or even a disc, by simply enclosing $A$ in a larger, convex region $A^{+}$such as the convex hull or circumcircle of $A$, generating a simulated realisation of $U$ in $A^{+}$, and clipping it to $A$.

\subsection{Extrapolation}

When extending a germ-grain model beyond the observation window, two cases may be distinguished, namely

(i) extending grains $Z_{i}^{*}$ such that $X_{i}^{*}+Z_{i}^{*}$ hits the boundary of $A$ based on $U \cap A$;

(ii) extending the pattern $U$ beyond the window $A$ based on $U \cap A$.

Below we discuss several geometric aspects in some generality. Specific aspects related to spatial cluster processes will be treated in subsequent sections.

For Poisson germ-grain models, the conditional distribution of $\left\{X_{i}^{*}+Z_{i}^{*}: i=1,2, \ldots\right\}$ given $\left\{\left(X_{i}^{*}+Z_{i}^{*}\right) \cap A: i=1,2, \ldots\right\}$ is such that the $X_{i}^{*}+Z_{i}^{*}$ are conditionally independent, and the conditional distribution of $X_{i}^{*}+Z_{i}^{*}$ depends only on $\left(X_{i}^{*}+Z_{i}^{*}\right) \cap A[20,48,50,71]$. Note that this conditional distribution as well as the law of $\left(X_{i}^{*}+Z_{i}^{*}\right) \cap A$ may have atoms, as for example if there is a non-zero probability that a single object $X_{i}^{*}+Z_{i}^{*}$ covers $A$ completely or in the conditional case, if a grain is specified fully by its restriction to $A$. Atoms need to be treated separately using integral-geometric factorisation techniques [79].

If the distribution governing $X$ is not that of a Poisson point process, as for spatial clustering problems, the grains can no longer be extended independently of each other. Other obstacles arise from the unobservability of the individual objects in the pattern (cf. section 2.2), and we need to extend grains based on the union set $U \cap A$. Sometimes, $U \cap A$ suffices to determine the individual sets $\left(X_{i}^{*}+Z_{i}^{*}\right) \cap A$; more often it will not be possible to determine the components uniquely from $U$ especially if the window $A$ is not convex or if objects may occlude one another. Indeed, the identification of the offspring partitioning is the whole point of spatial clustering.

To conclude this section, note that alternative classes of models include the various Poissonbased constructions described in [16], [80, chap. XIII], and Arak-Surgailis-Clifford mosaics and random graphs $[1,2,3]$. We use germ-grain models mainly because they are quite flexible while still remaining relatively simple from a computational point of view: Markov chain Monte Carlo simulation methods are available by combining existing methods for point processes and for Poisson processes of geometric objects, and parametric and nonparametric inferential methods can be carried over from existing methods for spatial point processes. Moreover, in the alternative models listed above, the geometric features may be connected (e.g. several line segments may have a common endpoint) in a fashion which is inappropriate to most of the applications considered here, although positively desirable for other applications such as random tessellations.

\section{Spatial Cluster PRocesses}

The identification of centres of clustering is of interest in many areas of applications, including archeology [37], mining [5, 16] and animal territory research [13]. In disease mapping the 
identification of cluster centres is of prime interest [62] and mine field detection relies on separating clusters of land mines from clutter of other kinds [19, 21].

Most traditional clustering approaches build a tree based on some similarity measure (see e.g. $[14,28,35,39,43,61]$ or other textbooks on multivariate statistics). From this tree, the number of clusters and the corresponding partition is decided in an ad hoc (and mostly subjective) manner. More recently, model based clustering techniques [21, 24] consider finite mixture models. The number of groups is determined by a Bayes factors or AIC criterium, and given the number of mixture components, model parameters are estimated by maximum likelihood, often using a variant of the EM algorithm. Most applications also allow a 'do not know' class for outliers or noise. The cluster centres only play an implicit role - approximated by the centre of gravity, principal axis or other 'mean' of the detected clusters - if they appear at all. Notable exceptions are $[58,59,60]$ who model uncertainty in point locations by means of a cluster process consisting of at most a single point, and [55] who employ variational analysis in the space of intensity measures of the parent point process.

In contrast, following up on earlier work $[6,54,52]$, this paper advocates the use of point process and germ-grain models (see section 2.1). A virtue of this approach is that the number of clusters, the locations of their centres, and the grouping or labelling of observed points into clusters, are intrinsic aspects of the underlying process (rather than additional parameters) and are all treated simultaneously. The most general model we consider is the independent cluster process introduced in section 3.1, but most attention will be focussed on the computationally convenient Cox cluster processes (section 3.2). The cluster formation densities are derived in section 3.3 below.

\subsection{Independent cluster processes}

Let $X$ be a point process on $\mathbb{R}^{d}$ and associate with each $X_{i}$ a finite cluster $Z_{i}$ of points 'centred' at the origin of $\mathbb{R}^{d}$. Throughout we will assume that the grains $Z_{i}$ are conditionally independent. The union of offspring $U=\cup_{i}\left(X_{i}+Z_{i}\right)$ is an independent cluster process [20, pp. 236-238], [17, pp. 75-81, 148 ff.]. Technical conditions of finiteness and measurability must be satisfied for such a process to exist, see [20, p. 236].

The data consist of a realisation of $Y=U \cap A$ in a compact window $A \subseteq \mathbb{R}^{d}$ of positive volume. Thus,

$$
\mathbf{y}=\left\{y_{1}, \ldots, y_{m}\right\}, \quad m>0, \quad \mathbf{y} \subseteq A
$$

is a configuration of daughters in $A$. The above formulation is quite flexible, in that it retains the possibility of locating putative cluster parents outside the window $A$ to counteract sampling bias effects (see the discussion in section 2.3) and of grain characteristics such as the daughter intensity or the spread of the cluster to be randomly and spatially varying.

In order to be able to base inference on penalised likelihoods, we shall restrict the germ process to lie inside some compact set $\mathcal{X} \subseteq \mathbb{R}^{d}$ of positive volume, and assume that its distribution is absolutely continuous with respect to a unit rate Poisson point process on $\mathcal{X}$. For each $\xi \in \mathcal{X}$ we are given the distribution $Q_{\xi}$ of a finite point process $Z_{\xi}$ on a compact subset $\tilde{\mathcal{X}}$ of $\mathbb{R}^{d} ; Z_{\xi}$ represents the offspring of a parent $\xi$ translated back to the origin to fit in the general germ-grain model of section 2.1. We assume that $Q_{\xi}$ is absolutely continuous with a density $g(\cdot \mid \xi)$ with respect to the distribution of a unit rate Poisson process on $\tilde{\mathcal{X}}$. Thus $\mathcal{Z}=\mathcal{N}=\mathcal{N}_{\tilde{\mathcal{X}}}$, the family of finite point configurations in $\tilde{\mathcal{X}}$. To ensure existence 
of $U$, we shall assume that the family of densities is jointly measurable seen as a function $g: \mathcal{X} \times \mathcal{N} \rightarrow \mathbb{R}^{+}$.

More generally, we could have set $\tilde{\mathcal{X}}=\mathbb{R}^{d}$ equipped with some finite diffuse intensity measure $\nu(\cdot)$, with the assumption that $Q_{\xi}$ is absolutely continuous with respect to the distribution of a Poisson process with intensity measure $\nu(\cdot)$. It is of interest to note that when $X$ is a Poisson process and we extend the process onto the whole of $\mathbb{R}^{d}$, then $Q$ may be almost surely reconstructed from a single joint observation of parents and daughters [10, 65].

Table 3.1 summarises standard nomenclature for special cases of the independent cluster model $[20,84]$.

\begin{tabular}{lll} 
Parents $X$ & Clusters $Z$ & Name of process $U$ \\
\hline general & general & Independent cluster process \\
Poisson & general & Poisson cluster process \\
general & Poisson & Cox cluster process \\
Poisson & Poisson & Neyman-Scott process \\
Poisson (homogeneous) & Poisson (uniform in disc) & Matérn cluster process \\
Poisson (homogeneous) & Poisson (Gaussian) & modified Thomas process \\
\hline
\end{tabular}

Table 1: Standard nomenclature for independent cluster processes.

\subsection{Cox cluster processes}

For simplicity, most attention will be focussed on the Cox cluster process model, where each grain $Z_{\xi}, \xi \in \mathcal{X}$, is a realisation of an inhomogeneous Poisson point process on $\tilde{\mathcal{X}}$ with intensity function $h(\cdot+\xi \mid \xi): \tilde{\mathcal{X}} \rightarrow[0, \infty)$. In other words, a parent point $\xi$ is replaced by a Poisson number of offspring with mean $H(\xi)=\int_{\tilde{\mathcal{X}}} h(t+\xi \mid \xi) d t \in(0, \infty)$, and given the number of offspring their locations are independently and identically distributed with probability density $f(\cdot)=h(\cdot \mid \xi) / H(\xi)$ on $\xi+\tilde{\mathcal{X}}$ (with respect to Lebesgue measure). We shall assume the intensity function $h(\cdot \mid \cdot)$ to be jointly measurable in its arguments, as well as integrable so that $H(\xi)<\infty$ for all $\xi \in \mathcal{X}$. As in $[21,52,54]$, scatter noise and outliers also known as orphans - are modelled by a Poisson point process of constant intensity $\epsilon>0$ independently of all $Z_{\xi}$. This fits into the germ-grain framework of section 2.1 by introducing an extra dummy or 'ghost' parent $x_{0}$. We shall write $h\left(\cdot \mid x_{0}\right) \equiv \epsilon$, and denote its integral over $\mathcal{X} \oplus \tilde{\mathcal{X}}$ by $H\left(x_{0}\right)$.

By the superposition property of Poisson processes, conditional on $X=\mathbf{x}=\left\{x_{1}, \ldots, x_{n}\right\}$, the combined offspring form a Poisson point process on $\mathcal{X} \oplus \tilde{\mathcal{X}}$ with intensity function

$$
\lambda(\cdot \mid \mathbf{x})=\epsilon+\sum_{i=1}^{n} h\left(\cdot \mid x_{i}\right)
$$

with the convention that $h\left(t \mid x_{i}\right)=0$ if $t \notin x_{i}+\tilde{\mathcal{X}}, i=1, \ldots, n$. The marginal distribution of $U$ is that of a Cox point process [84, p. 144].

Often, the intensity function $h(t \mid \xi)$ will depend only on the distance $d(\xi, t)$ between $\xi$ and 
t. An example is

$$
h(t \mid \xi)= \begin{cases}\mu & \text { if } d(\xi, t) \leq R_{h} \\ 0 & \text { otherwise }\end{cases}
$$

which, if $X$ is also a Poisson process, is known as the Matérn cluster process [64]. Another interesting special case is (for $d=2$, say)

$$
h(t \mid \xi)=\frac{\mu}{2 \pi \sigma^{2}} e^{-d(\xi, t)^{2} / 2 \sigma^{2}} .
$$

According to (3.3), the daughters follow an isotropic Gaussian distribution with centre $\xi$. Again if $X$ is a Poisson process, the distribution of $U$ is called the modified Thomas process. More generally, the spread $\sigma$ may depend on $\xi$. For further details, consult $[20,26]$ or [84].

For a Cox cluster process, conditional on $X=\mathbf{x}=\left\{x_{1}, \ldots, x_{n}\right\}$ and the number $m$ of offspring, the points are drawn independently from a finite mixture distribution [34, 87] with $n+1$ component distributions determined by the $x_{i}$ and weights

$$
p_{i}=\frac{H\left(x_{i}\right)}{\sum_{i=0}^{n} H\left(x_{i}\right)}, \quad i=0, \ldots, n .
$$

If the intensity function $h$ is translation invariant in the sense that $h(t+\xi \mid \xi)=h(t \mid 0)$ for all $\xi \in \mathcal{X}$ - a common assumption in our spatial context - the weights are identical for all parents except the ghost, a rather unnatural restriction in the finite mixture context. Furthermore, the connection with mixture distributions is lost when the clusters are no Poisson processes.

To conclude this section, note that some parents may be childless. In particular, if the clusters $Z_{\xi}$ are Poisson processes, they have a positive probability of being empty. If in a particular application there is no interest in such parents, one could condition each $Z_{\xi}$ on $\left\{Z_{\xi} \neq \emptyset\right\}$, or consider only those parents having at least one daughter.

\subsection{Cluster formation densities}

In order to be able to draw inference about parents and cluster membership, we need the (posterior) distribution of $W=\left\{\left(x_{0}, Z_{0}\right), \ldots,\left(X_{n}, Z_{n}\right)\right\}$, i.e. of parents $X_{i}$ marked by their associated grain $Z_{i}, i=0, \ldots, n$. We will take a Bayesian approach based on

$$
\begin{aligned}
p_{W \mid U}\left(\left\{\left(x_{i}, \mathbf{z}_{i}\right)\right\}_{i \leq n} \mid \mathbf{u}\right) & \propto P\left(\mathbf{z}_{0}, \ldots, \mathbf{z}_{n} \mid x_{0}, \ldots, x_{n}, \mathbf{u}\right) p_{X \mid U}(\mathbf{x} \mid \mathbf{u}) \\
& =c(\mathbf{u}) P\left(\mathbf{z}_{0}, \ldots, \mathbf{z}_{n} \mid x_{0}, \ldots, x_{n}, \mathbf{u}\right) p_{U \mid X}(\mathbf{u} \mid \mathbf{x}) p_{X}(\mathbf{x}),
\end{aligned}
$$

the posterior density of $W$ with respect to a unit rate Poisson process on $\mathcal{X}$ marked at $\xi \in \mathcal{X}$ by a label in $\mathcal{P}(\mathbf{u}-\xi)$ according to the uniform distribution on the power set of $\mathbf{u}$ translated back to the origin. The term $p_{X}(\mathbf{x})$ is the prior density for $X$ with respect to the distribution of a unit rate Poisson process on $\mathcal{X}$, and $c(\mathbf{u})$ a normalising constant depending on the 'data' u. If only the cluster centres are of interest, the posterior density of $X$ (with respect to the distribution of a unit rate Poisson process on $\mathcal{X}$ ) may be used instead:

$$
p_{X \mid U}(\mathbf{x} \mid \mathbf{u})=c^{\prime}(\mathbf{u}) p_{U \mid X}(\mathbf{u} \mid \mathbf{x}) p_{X}(\mathbf{x}) .
$$

We will discuss the choice of prior later on and here describe only the 'forward terms' of cluster formation. 
Firstly, recall from section 3.1 that conditional on $X=\mathbf{x}=\left\{x_{1}, \ldots, x_{n}\right\}$, the grains $Z_{1}, \ldots, Z_{n}$ associated with $x_{1}, \ldots, x_{n}$ respectively are independent with distributions that are absolutely continuous with respect to a unit rate Poisson process on $\tilde{\mathcal{X}}$. Thus, the conditional joint density of $\left(Z_{1}, \ldots, Z_{n}\right)$ equals

$$
\prod_{i=1}^{n} g\left(\mathbf{z}_{i} \mid x_{i}\right)
$$

with respect to the $n$-fold product measure of unit rate Poisson processes on $\tilde{\mathcal{X}}$. The orphans $Z_{0}$ are modelled as a Poisson process of rate $\epsilon>0$. Again conditioning on $X=\mathbf{x}$, the superposition $U$ is absolutely continuous with respect to the distribution of a unit rate Poisson process on $\mathcal{X} \oplus \tilde{\mathcal{X}}$. Its density at $\mathbf{u}=\left\{u_{1}, \ldots, u_{m}\right\}$ can be found by summing over all partitions in sibling clusters

$$
\begin{aligned}
& p_{U \mid X}(\mathbf{u} \mid \mathbf{x})= \\
& \quad e^{(1-\epsilon)|\mathcal{X} \oplus \tilde{\mathcal{X}}|-n|\tilde{\mathcal{X}}|} \sum_{\varphi} \epsilon^{n\left(\mathbf{u}_{\varphi^{-1}(\{0\})}\right)} \prod_{i=1}^{n} g\left(\mathbf{u}_{\varphi^{-1}(\{i\})}-x_{i} \mid x_{i}\right) \mathbf{1}\left\{\mathbf{u}_{\varphi^{-1}(\{i\})}-x_{i} \subseteq \tilde{\mathcal{X}}\right\}
\end{aligned}
$$

where the sum ranges over all possible offspring-to-parent assignment functions $\varphi:\{1, \ldots, m\}$ $\rightarrow\{0, \ldots, n\}, \mathbf{u}_{\varphi^{-1}(\{i\})}=\left\{u_{j}: \varphi(j)=i\right\}$ consists of those $u_{j}$ ascribed to parent $x_{i}$ by $\varphi$, and $n(\cdot)$ denotes cardinality. Equation (3.6) is most readily derived using Janossy densities [20, p. 122]. The details can be found in [52, Lemma 23]. Note that (3.6) can be expressed as

$$
e^{(1-n-\epsilon)|\mathcal{X} \oplus \tilde{\mathcal{X}}|} \sum_{\varphi} \epsilon^{n\left(\mathbf{u}_{\varphi^{-1}(\{0\})}\right)} \prod_{i=1}^{n} g^{\prime}\left(\mathbf{u}_{\varphi^{-1}(\{i\})}-x_{i} \mid x_{i}\right)
$$

where $g^{\prime}\left(\cdot-x_{i} \mid x_{i}\right)=e^{|\mathcal{X} \oplus \tilde{\mathcal{X}}|-|\tilde{\mathcal{X}}|} g\left(\cdot-x_{i} \mid x_{i}\right) \mathbf{1}\left\{\cdot-x_{i} \subseteq \tilde{\mathcal{X}}\right\}$ is a density of the translated typical grain with respect to a unit rate Poisson process on $\mathcal{X} \oplus \tilde{\mathcal{X}}$.

Next, consider the conditional distribution of the complete model given the cluster centres $x_{1}, \ldots, x_{n}$. Since we already derived the conditional joint density of $\left(Z_{1}, \ldots, Z_{n}\right)$, an identification

$$
\left(\mathcal{Z}^{n}, \pi_{\mathcal{Z}}^{n}, \mathcal{A}\right) \leftrightarrow\left(\mathcal{N}_{\mathcal{X} \times \mathcal{Z}}, \xi_{\mathbf{x}}, \mathcal{B}\right)
$$

of grain vectors $\left(\mathbf{z}_{1}, \ldots, \mathbf{z}_{n}\right) \in \mathcal{Z}^{n}$ with the marked point configuration $\left\{\left(x_{1}, \mathbf{z}_{1}\right), \ldots,\left(x_{n}, \mathbf{z}_{n}\right)\right\}$ $\in \mathcal{N}_{\mathcal{X} \times \mathcal{Z}}$ is needed. Here $\mathcal{Z}=\mathcal{N}_{\tilde{\mathcal{X}}}$ is the grain space (cf. section 2.1) consisting of all finite point configurations, $\pi_{\mathcal{Z}}^{n}$ is the $n$-fold product measure of unit rate Poisson processes on $\tilde{\mathcal{X}}$, $\mathcal{A}$ is the usual Borel product $\sigma$-algebra of the weak topology [20], and $\mathcal{B}$ the Borel $\sigma$-algebra of the weak topology on marked point patterns. To do so, define a measurable bijection $i_{\mathbf{x}}$ (in the sense that the complement of the range of $i_{\mathbf{x}}$ has measure zero under $\xi_{\mathbf{x}}$ ) depending on the parent pattern $\mathbf{x}=\left\{x_{1}, \ldots, x_{n}\right\}$ by

$$
i_{\mathbf{x}}:\left(\mathbf{z}_{1}, \ldots, \mathbf{z}_{n}\right) \mapsto\left\{\left(x_{1}, \mathbf{z}_{1}\right), \ldots\left(x_{n}, \mathbf{z}_{n}\right)\right\} .
$$

Using the identification thus defined, the measure $\xi_{\mathbf{x}}$ is given by $\xi_{\mathbf{x}}(B)=\pi_{\mathcal{Z}}^{n}\left(i_{\mathbf{x}}^{-1}(B)\right)$ for all $B \in \mathcal{B}$. 
Finally, the conditional distribution of $W$ or equivalently the marks $Z_{i}$ given $(X, U)$ is discrete, with probabilities

$$
\begin{aligned}
& P\left(\mathbf{z}_{0}, \ldots \mathbf{z}_{n} \mid x_{0}, \ldots, x_{n}, \mathbf{u}\right)= \\
& \frac{\epsilon^{n\left(\mathbf{z}_{0}\right)} \prod_{i=1}^{n} g\left(\mathbf{z}_{i} \mid x_{i}\right)}{\sum_{\varphi} \epsilon^{n\left(\mathbf{u}_{\varphi^{-1}(\{0\})}\right)} \prod_{i=1}^{n} g\left(\mathbf{u}_{\varphi^{-1}(\{i\})}-x_{i} \mid x_{i}\right) \mathbf{1}\left\{\mathbf{u}_{\varphi^{-1}(\{i\})}-x_{i} \subseteq \tilde{\mathcal{X}}\right\}}
\end{aligned}
$$

provided the union $\cup_{i}\left(x_{i}+\mathbf{z}_{i}\right)$ equals $\mathbf{u}$. If $g(\cdot-\xi \mid \xi)$ is hereditary (cf. section 4.1) for each $\xi \in \mathcal{X}$, the sum in the denominator of (3.7) over all functions $\varphi$ ascribing parents to each offspring, is non-zero. Otherwise, we have to impose the condition that the grain partition and $(X, U)$ are compatible, in the sense that there exists at least one $\varphi$ for which the term $\epsilon^{n\left(\mathbf{u}_{\varphi^{-1}(\{0\})}\right)} \prod_{i=1}^{n} g\left(\mathbf{u}_{\varphi^{-1}(\{i\})}-x_{i} \mid x_{i}\right) \mathbf{1}\left\{\mathbf{u}_{\varphi^{-1}(\{i\})}-x_{i} \subseteq \tilde{\mathcal{X}}\right\}$ is strictly positive [52, Theorem 29].

For Cox cluster processes, the formulae (3.4)-(3.7) can be greatly simplified. Since $Z_{\xi}$ has density

$$
g(\mathbf{z} \mid \xi)=\exp \left[\int_{\tilde{\mathcal{X}}}(1-h(t+\xi \mid \xi)) d t\right] \prod_{z \in \mathbf{z}} h(z+\xi \mid \xi)
$$

with respect to a unit rate Poisson process on $\tilde{\mathcal{X}},(3.6)$ reduces to

$$
\begin{aligned}
p_{U \mid X}(\mathbf{u} \mid \mathbf{x}) & =\exp \left[\int_{\mathcal{X} \oplus \tilde{\mathcal{X}}}(1-\lambda(t \mid \mathbf{x})) d t\right] \sum_{\varphi} \prod_{i=0}^{n} \prod_{t \in \mathbf{u}_{\varphi^{-1}(\{i\})}} h\left(t \mid x_{i}\right) \\
& =\exp \left[\int_{\mathcal{X} \oplus \tilde{\mathcal{X}}}(1-\lambda(t \mid \mathbf{x})) d t\right] \prod_{j=1}^{m} \lambda\left(u_{j} \mid \mathbf{x}\right)
\end{aligned}
$$

coding $h\left(\cdot \mid x_{0}\right) \equiv \epsilon$ for the dummy parent $x_{0}$. Thus, (3.8) is in accordance with the fact that the independent superposition of Poisson processes is again a Poisson process, here with intensity $\lambda(\cdot \mid \mathbf{x})$ (cf. (3.1) and the discussion in section 3.2).

As for the offspring labelling, (3.7) for a Cox cluster process equals

$$
P\left(\mathbf{z}_{0}, \ldots, \mathbf{z}_{n} \mid x_{0}, \ldots, x_{n}, \mathbf{u}\right)=\frac{\prod_{i=0}^{n} \prod_{t \in \mathbf{z}_{i}} h\left(t+x_{i} \mid x_{i}\right)}{\prod_{j=1}^{m} \lambda\left(u_{j} \mid \mathbf{x}\right)}
$$

whenever $\cup_{i}\left(x_{i}+\mathbf{z}_{i}\right)=\mathbf{u}$, see [54] or [52, Corollary 30].

In terms of the label allocation function $\varphi:\{1, \ldots, m\} \mapsto\{0,1, \ldots, n\}$ allocating each daughter point to its parent, equation (3.9) implies that the daughters are ascribed to a cluster centre $x_{I}$ independently of one another, with probabilities

$$
P(\varphi(j)=I)=\frac{h\left(u_{j} \mid x_{I}\right)}{\lambda\left(u_{j} \mid \mathbf{x}\right)} .
$$

The analogue of this result for finite mixtures with $m$ and $n$ fixed was called the Random Imputation Principle by Diebolt and Robert [24]. It was taken as an assumption by Binder $[12$, p. 32, above (2.1)]. Note the statement holds only for Cox cluster processes, i.e. when the clusters are Poisson. 


\section{BAYESIAN CLUSTER ANALYSIS}

From section 2.2, recall that the prime object of spatial cluster analysis is to evaluate conditional expectations of quantities such as the number of clusters and the mean number of points per cluster based on the posterior distribution (3.4) of the complete data $W$ given y. In the previous section, we derived the densities associated with cluster formation. In section 4.1 below, we discuss the prior, and investigate properties of the posterior distribution in section 4.2. Then we turn to the the problems of generating realisations of (3.4) by Markov chain Monte Carlo methods, and of estimating the model parameters (sections 4.3section 4.4). In section 4.5, we propose an adaptive coupling from the past algorithm that yields exact samples from (3.4). Throughout, the redwood data set $[72,85]$ is used as an illustration.

\subsection{Markov point processes}

In this section we focus on the prior term $p_{X}(\mathbf{x})$ in $(3.4)$, which we shall assume to be the density of a Markov point process. Accessible introductions to Markov point processes can be found in [17, pp. 155-159] and [76]. Theory and properties are set out in [20, pp. 124-125, $573 \mathrm{ff}]$, [71, pp. 189-193], [9, 68, 75, 77, 78]. A recent textbook is [53]. Following is a brief summary of the facts we need.

Let $X$ be a point process on a compact subset $\mathcal{X} \subseteq \mathbb{R}^{d}$ of positive volume, whose distribution is absolutely continuous with respect to a unit rate Poisson process on $\mathcal{X}$, say with density $p_{X}(\cdot)$. Then $X$ is Markov at range $R$ in the sense of Ripley and Kelly [77] if the ratio

$$
\lambda_{X}(\xi ; \mathbf{x})=\frac{p_{X}(\mathbf{x} \cup\{\xi\})}{p_{X}(\mathbf{x})}
$$

is well-defined for all $\xi \in \mathcal{X}$ (i.e. $p_{X}(\mathbf{x} \cup\{\xi\})=0$ implies $p_{X}(\mathbf{x})=0$; in this case we will also say that $p_{X}(\cdot)$ is hereditary) and depends only on those $x_{i} \in \mathbf{x}$ for which $d\left(x_{i}, \xi\right) \leq R$. More generally, the fixed range dependence may be replaced by an arbitrary symmetric neighbourhood relation $\sim$ (so that (4.1) depends on $x_{i} \sim \xi$ only). Even more general Markov point processes are considered in [9], and the Markovianity of spatial cluster processes is studied in [8]. A (Markov) point process defined by its density with respect to a unit rate Poisson process is said to be locally stable if its conditional intensity (4.1) is well-defined and uniformly bounded in both its arguments.

To model patterns in which the points tend to avoid coming too close together, it is convenient to consider pairwise-interaction processes with densities of the form

$$
p_{X}(\mathbf{x})=\alpha \prod_{x \in \mathbf{x}} \beta(x) \prod_{x \sim x^{\prime} \in \mathbf{x}} \gamma\left(x, x^{\prime}\right)
$$

where $\beta: \mathcal{X} \rightarrow[0, \infty)$ (the 'intrinsic activity') and $\gamma: \mathcal{X} \times \mathcal{X} \rightarrow[0, \infty)$ (the 'pairwise interaction') are measurable functions, $\gamma$ is symmetric, and $\alpha>0$ is the normalising constant. This model is well-defined (i.e. the density is integrable) at least whenever $\beta(\cdot)$ is uniformly bounded and $\gamma(\cdot, \cdot) \leq 1$.

A standard example of (4.2) is the Strauss process [85] with $\beta(\cdot) \equiv \beta>0$ and

$$
\gamma\left(x, x^{\prime}\right)= \begin{cases}\gamma & \text { if } d\left(x, x^{\prime}\right) \leq R \\ 1 & \text { otherwise }\end{cases}
$$


where $0 \leq \gamma \leq 1$, which has density

$$
p_{X}(\mathbf{x})=\alpha \beta^{n(\mathbf{x})} \gamma^{s(\mathbf{x})}
$$

where $n(\mathbf{x})$ is the number of points in $\mathbf{x}$ and $s(\mathbf{x})$ is the number of pairs $x, x^{\prime}$ with $d\left(x, x^{\prime}\right) \leq R$. The model favours realisations $\mathbf{x}$ that tend to have more points at distances larger than $R$ than under the Poisson model, that is there is repulsion between the points. The special case $\gamma=0$ in which no $R$-close point pairs are permitted is known as the hard core process; $\gamma=1$ corresponds to a Poisson process with intensity $\beta$.

More formally, a point process density $p_{X}(\cdot)$ is called anti-monotone (or repulsive) if

$$
\lambda_{X}\left(\xi ; \mathbf{x}^{\prime}\right) \leq \lambda_{X}(\xi ; \mathbf{x})
$$

for all $\xi$ whenever $\mathbf{x} \subseteq \mathbf{x}^{\prime}$ and monotone (or attractive) if its conditional intensity satisfies

$$
\lambda_{X}\left(\xi ; \mathbf{x}^{\prime}\right) \geq \lambda_{X}(\xi ; \mathbf{x}) .
$$

The reader may verify that the Strauss process is repulsive for all $\gamma \leq 1$.

\subsection{Sampling bias for independent cluster processes}

Note that the restriction $Y$ of an independent cluster process $U$ to some compact observation window $A$ is itself an independent cluster process. Indeed,

$$
Y=U \cap A=\bigcup_{x \in X}\left(x+Z_{x}\right) \cap A=\bigcup_{x \in X}\left(x+\left(Z_{x} \cap(A-x)\right)\right) .
$$

The distribution $Q_{\xi, A}$ of the grain $Z_{\xi} \cap(A-\xi)$ associated to $\xi$ in the $A$-clipped process has density

$$
g_{A}(\mathbf{z} \mid \xi)=\sum_{k=0}^{\infty} \frac{1}{k !} \int \cdots \int_{(\tilde{\mathcal{X}} \backslash(A-\xi))^{k}} g\left(\mathbf{z} \cup\left\{v_{1}, \ldots, v_{k}\right\} \mid \xi\right) d v_{1} \ldots d v_{k}
$$

with respect to a unit rate Poisson process on $\tilde{\mathcal{X}}$. It follows that the posterior distribution of $X$ given $Y$ is analogous to (3.5), except for the fact that $g_{A}(\cdot \mid \cdot)$ features instead of $g(\cdot \mid \cdot)$. As before, a ghost parent is added to account for scatter noise.

For Cox cluster processes, (4.4) simplifies to

$$
g_{A}(\mathbf{z} \mid \xi)=\exp \left[\int_{\tilde{X}}(1-h(t+\xi \mid \xi) \mathbf{1}\{t \in A-\xi\}) d t\right] \prod_{z \in \mathbf{z}} h(z+\xi \mid \xi)
$$

for $\mathbf{z} \subseteq A-\xi$, the density of a Poisson point process with intensity function $h(\cdot+\xi \mid \xi) \mathbf{1}\{\cdot \in$ $A-\xi\}$. Hence, conditionally on $X=\mathbf{x}=\left\{x_{1}, \ldots, x_{n}\right\}, Y$ is an inhomogeneous Poisson process on $A$ with intensity function

$$
\lambda(a \mid \mathbf{x})=\epsilon+\sum_{i=1}^{n} h\left(a \mid x_{i}\right), \quad a \in A
$$

where $\epsilon>0$ is the background clutter term (cf. section 3.2). As for the prior, one could simply assume the parents to be distributed as a Poisson point process, but it seems more 
natural to incorporate repulsion at short range to avoid 'over fitting' in the sense of many close parents. Thus, one might take as prior for example a hard core process (cf. section 4.1) with density

$$
p_{X}(\mathbf{x})= \begin{cases}\alpha \beta^{n(\mathbf{x})} & \text { if } d\left(x_{i}, x_{j}\right)>R \text { for all pairs } \\ 0 & \text { otherwise }\end{cases}
$$

with respect to a unit rate Poisson process on $\mathcal{X}$.

Upon observing $Y=\mathbf{y}=\left\{y_{1}, \ldots, y_{m}\right\}$, the analogue of (3.5) for the $A$-clipped process is

$$
p_{X \mid Y}(\mathbf{x} \mid \mathbf{y})=c(\mathbf{y}) p_{X}(\mathbf{x}) \exp \left[\int_{A}(1-\lambda(a \mid \mathbf{x})) d a\right] \prod_{j=1}^{m} \lambda\left(y_{j} \mid \mathbf{x}\right)
$$

which has posterior conditional intensity

$$
\lambda_{X \mid Y}(\xi ; \mathbf{x} \mid \mathbf{y})=\lambda_{X}(\xi ; \mathbf{x}) \exp \left[-\int_{A} h(a \mid \xi) d a\right] \prod_{j=1}^{m}\left[1+\frac{h\left(y_{j} \mid \xi\right)}{\lambda\left(y_{j} \mid \mathbf{x}\right)}\right] .
$$

If the prior density $p_{X}(\cdot)$ is that of a repulsive Markov point process, the posterior distribution specified by (4.6) is hereditary and repulsive too. The posterior range of interaction depends on the supports of the family $h(\cdot \mid \xi), \xi \in \mathcal{X}$, of intensity functions. If furthermore the prior density $p_{X}(\cdot)$ is locally stable with bound $\lambda$ for its conditional intensity and $h(\cdot \mid \cdot)$ is uniformly bounded in both its arguments by $H$, then $\lambda_{X \mid Y}(\xi ; \mathbf{x} \mid \mathbf{y}) \leq \lambda(1+H / \epsilon)^{m}$ implying local stability of (4.6).

\subsection{Spatial birth-and-death processes}

In this section, we address the problem of sampling from the posterior distribution of the complete data $W$ given partial observations $Y=U \cap A=\left\{y_{1}, \ldots, y_{m}\right\}$ of a Cox cluster process $U$ within some compact observation window $A$. Note that since the offspring allocation labels are discrete and distributed according to (3.9), and by the Poisson assumption any daughters in $A^{c}$ are conditionally independent of those in $A$, the problem reduces to sampling from the conditional distribution (4.6) of $X$ given $Y$. Since direct sampling does not seem feasible, we apply Markov chain Monte Carlo techniques. Perhaps the oldest such technique is based on spatial birth-and-death processes [69], continuous time Markov processes whose transitions are either births or deaths. The traditional choice $[9,66,72]$ is to take a birth rate proportional to the posterior conditional intensity and a constant death rate. Under mild non-explosion conditions, this procedure converges to the target distribution and hence yields approximate samples if run for long enough [66,69]. A disadvantage is that the total birth rate is difficult to compute, and the product over data points in (4.7) may be very large. For these reasons, we prefer to work with the alternative birth rate

$$
b(\mathbf{x}, \xi)=\lambda_{X}(\xi ; \mathbf{x})\left[1+\sum_{j=1}^{m} \frac{h\left(y_{j} \mid \xi\right)}{\epsilon}\right]
$$

which is less peaked than the posterior conditional intensity, while retaining the desirable property of placing most new-born points in the vicinity of points of $\mathbf{y}$. In order to satisfy 
the detailed balance equations

$$
p_{X \mid Y}(\mathbf{x} \mid \mathbf{y}) b(\mathbf{x}, \xi)=p_{X \mid Y}(\mathbf{x} \cup\{\xi\} \mid \mathbf{y}) d(\mathbf{x} \cup\{\xi\}, \xi),
$$

the death rate for deleting $\xi$ from configuration $\mathbf{x} \cup\{\xi\}$ is

$$
d(\mathbf{x} \cup\{\xi\}, \xi)=\frac{\exp \left[\int_{A} h(a \mid \xi) d a\right]}{\prod_{j=1}^{m}\left[1+\frac{h\left(y_{j} \mid \xi\right)}{\lambda\left(y_{j} \mid \mathbf{x}\right)}\right]}\left[1+\sum_{j=1}^{m} \frac{h\left(y_{j} \mid \xi\right)}{\epsilon}\right] .
$$

Note that for any locally stable prior distribution for which $\lambda_{X}(\xi ; \mathbf{x}) \leq \lambda$ uniformly in $\mathbf{x}$ and $\xi$, and any $h(\cdot \mid \cdot)$ that is uniformly bounded in both its arguments by $H$, the total birth rate

$$
B(\mathbf{x})=\int_{\mathcal{X}} b(\mathbf{x}, \xi) d \xi \leq \lambda\left[|\mathcal{X}|+\frac{1}{\epsilon} \sum_{j=1}^{m} \int_{\mathcal{X}} h\left(y_{j} \mid \xi\right) d \xi\right]:=B
$$

is bounded from above by a constant $B \leq \lambda|\mathcal{X}|(1+m H / \epsilon)$ that is easy to evaluate for typical choices of $h(\cdot \mid \cdot)$ such as (3.2) or (3.3). The total death rate from parent configuration $\mathbf{x}$ satisfies

$$
D(\mathbf{x})=\sum_{i} d\left(\mathbf{x}, x_{i}\right) \geq n(\mathbf{x})(1+H / \epsilon)^{-m} .
$$

Hence, by the Preston theorem [68] (see e.g. [9, 66]), there exists a unique spatial birthand-death process with transition rates given by (4.8) and (4.9). It has unique equilibrium distribution $p_{X \mid Y}(\cdot \mid \mathbf{y})$, to which it converges in distribution from any initial state.

From an algorithmic point of view, if the current state is $\mathbf{x}$, after an exponentially distributed sojourn time of rate $B+D(\mathbf{x})$, with probability $D(\mathbf{x}) /(B+D(\mathbf{x}))$ a point of $\mathbf{x}$ is deleted according to the distribution $d\left(\mathbf{x}, x_{i}\right) / D(\mathbf{x})$; a birth is proposed with the complementary probability $B /(B+D(\mathbf{x}))$ by sampling a candidate $\xi$ from the mixture density $\frac{\lambda}{B}\left[1+\sum_{j=1}^{m} \frac{h\left(y_{j} \mid \xi\right)}{\epsilon}\right]$, which is then accepted with probability $\lambda_{X}(\xi ; \mathbf{x}) / \lambda$.

4.3.1 Example: redwood seedlings Figure 1 (left) shows the locations of 62 redwood seedlings in a square of side approximately $23 \mathrm{~m}$. The data were extracted by Ripley [72] from a larger data set in Strauss [85]. The K-function [73, 74] for these data is given in [72] and suggests aggregation. As noted by Strauss this is caused by the presence of stumps known to exist in the plot, but whose position has not been recorded.

Previous analyses of this data set include that of Strauss, who fitted a model later criticised by Kelly and Ripley [44]. Ripley [72] concluded we should reject the Poisson hypothesis and remarked that there appears to be both clustering and inhibition between clusters.

Diggle [26] fitted a Poisson cluster process of Thomas type and reported least squares estimates $(25.6,0.042)$ for the parent intensity and the standard deviation of daughterparent distances. A goodness of fit test showed adequate fit, but, from a biological point of view, a mean number of 26 stumps seems implausible. In [25], a Poisson cluster process of Matérn type was fitted with similar results (radius 0.061 and 29 clusters). None of the 

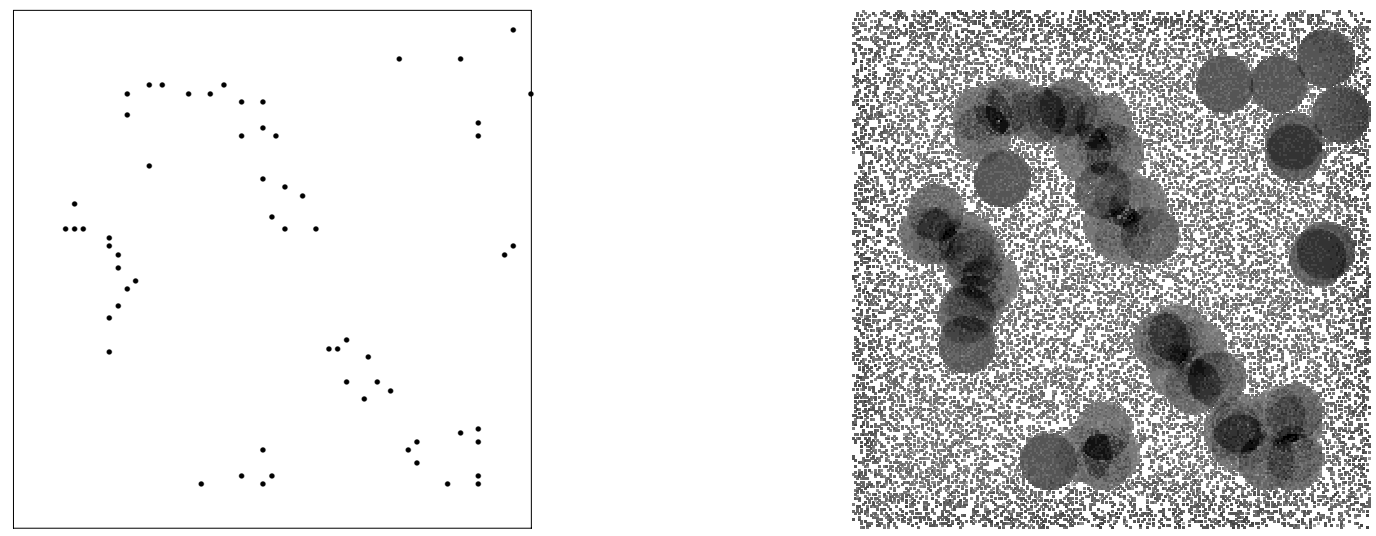

Figure 1: (Left) Positions of 62 redwood seedlings in a unit square and (right) log posterior parent intensity surface based on a Cox-Matérn cluster process with $R_{h}=0.061$ and on average 2.14 points per cluster, noise intensity $\epsilon=10.0$ and a hard core prior with $R=0.03$ and $\beta=1.0$ by spatial birth-and-death over $2.0 \times 10^{4}$ time units.

above have looked at cluster centre location. This was first studied in [6] and by Lawson [51] who fitted a Poisson-Thomas cluster process and reported 16 parents. An approach based on variational methods can be found in [55].

In earlier work $[6,52,54]$, we analysed the redwood data using a modified Thomas displacement function (3.3) and a Strauss prior (4.3) with interaction distance 0.084 [26] and $\log \beta=\log \gamma=-10$. Simulation was based on a constant death rate spatial birth-and-death process. Initialising with parameter values $\mu=7, \sigma=0.042$ and an empty list of cluster centres, we ran the birth-and-death process for 2 time units and found maximum likelihood estimates $\mu=6.5$ and $\sigma=0.05$.

Here, we use the spatial birth-and-death process with rates (4.8)-(4.9) to sample from the posterior distribution of cluster centres for a Cox model with a Matérn style intensity function given by (3.2) with $R_{h}=0.061$ and $\mu=2.14 /\left(\pi R_{h}^{2}\right)$ as in [25], orphan intensity $\epsilon=10.0$, and a hard core prior with $R=0.03$ and $\beta=1.0$. The posterior intensity surface of parents in $\mathcal{X}=\left[-R_{h}, 1.0+R_{h}\right]^{2}$ over $2.0 \times 10^{4}$ time units after a burn-in period of 200.0 units with empty initial state is plotted in figure 1 (right); for the posterior histogram of the number of clusters, see figure 2 (right). To indicate the effect of the choice of parameters, the posterior histogram for $\beta=0.052$ and an average cluster size of 4.3 is shown in figure 2 (left). It can be seen that the latter choice shifts the posterior histogram towards fewer cluster centres. 

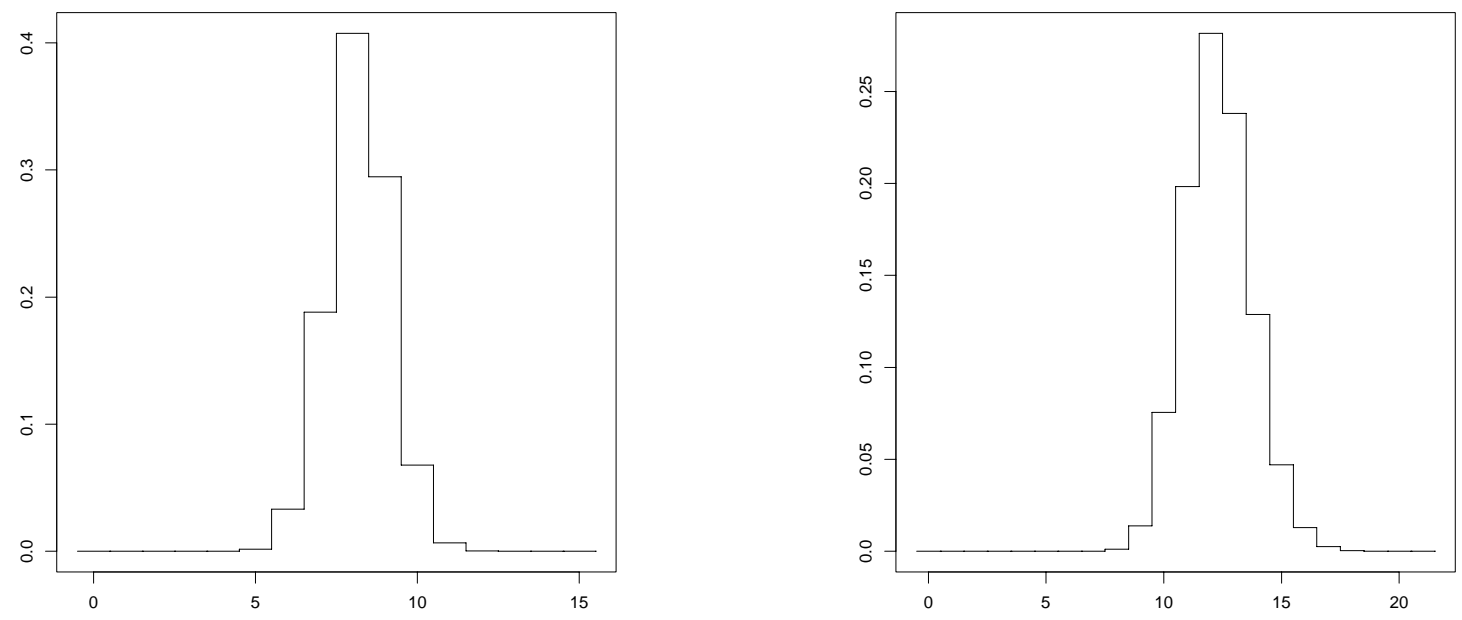

Figure 2: (Left) Posterior histogram for the number of parents given the data of figure 1 (left) based on a Cox-Matérn cluster process with $R_{h}=0.061$ and on average 4.3 points per cluster, noise intensity $\epsilon=10.0$ and a hard core prior with $R=0.03$ and $\beta=\exp (4.3+2.0 \log (\epsilon /(\epsilon+\mu))$ by spatial birth-and-death over $2.0 \times 10^{5}$ time units and (right) for on average 2.14 points and $\beta=1.0$ over $2.0 \times 10^{4}$ time units as in figure 1 (right).

\subsection{Parameter estimation}

In general, the independent cluster model $g(\cdot \mid \cdot)$ will contain parameters $\theta$ that must be estimated. For the Cox cluster model, the parameters are the clutter intensity $\epsilon$ as well as parameters of the displacement function $h(\cdot \mid \cdot)$ specifying the shape, the spread and the number of daughters in each cluster. Moreover, the prior model $p_{X}(\cdot)$ also contains parameters, but since these are merely used as regularisation to avoid over fitting, we will treat these as fixed.

We shall use the Monte Carlo maximum likelihood method for missing data models [29], see also [30]. In the context of detecting the centres in an independent cluster process, the observed data consists of a point pattern $\mathbf{y}$, the combined offspring in the window $A$. The missing data are both the parents and their associated grains. In terms of the cluster formation density derived in section 3.3 , the $\log$ likelihood ratio with respect to a fixed reference parameter $\theta_{0}$ can be written as

$$
l(\theta)=\log E_{\theta_{0}}\left[\prod_{i=0}^{n(X)} \frac{g_{\theta}\left(Z_{i} \mid X_{i}\right)}{g_{\theta_{0}}\left(Z_{i} \mid X_{i}\right)} \mid Y=\mathbf{y}\right]
$$

by importance sampling theory. The Monte Carlo analogue $l_{k}(\theta)$ of (4.10) is obtained by 
replacing the expectation by the average in a sample $W_{1}, \ldots, W_{k}$ from the complete model under the conditional distribution with parameter $\theta_{0}$. Differentiating with respect to $\theta$, the parameter of interest, we obtain

$$
\nabla l_{k}(\theta)=\frac{1}{k} \sum_{j=1}^{k} w_{j, \theta_{0}, \theta} \frac{\nabla\left[\prod_{\left(X_{i}, Z_{i}\right) \in W_{j}} g_{\theta}\left(Z_{i} \mid X_{i}\right)\right]}{\prod_{\left(X_{i}, Z_{i}\right) \in W_{j}} g_{\theta}\left(Z_{i} \mid X_{i}\right)}
$$

where $w_{j, \theta_{0}, \theta}=\left(\prod_{\left(X_{i}, Z_{i}\right) \in W_{j}} \frac{g_{\theta}\left(Z_{i} \mid X_{i}\right)}{g_{\theta_{0}}\left(Z_{i} \mid X_{i}\right)}\right) /\left(\frac{1}{k} \sum_{j=1}^{k} \prod_{\left(X_{i}, Z_{i}\right) \in W_{j}} \frac{g_{\theta}\left(Z_{i} \mid X_{i}\right)}{g_{\theta_{0}}\left(Z_{i} \mid X_{i}\right)}\right)$ are the importance weights. The well-known EM algorithm [23] is an iterative procedure based on (4.11) that consists of two steps: the E-step computes the conditional log likelihood given the data and current estimates of the parameters, the M-step maximises the result with respect to the parameter. Thus, the importance weights reduce to 1 , but resampling is needed at each step. For a critical evaluation of these and other parameter estimation methods, the reader is referred to [30], see also [27, 31, 38].

For Cox cluster processes, (4.10) simplifies to

$$
l(\theta)=\log E_{\theta_{0}}\left[e^{\sum_{i=0}^{n(X)}\left(H_{\theta_{0}}\left(X_{i}\right)-H_{\theta}\left(X_{i}\right)\right)} \prod_{i=0}^{n(X)} \prod_{t \in X_{i}+Z_{i}} \frac{h_{\theta}\left(t \mid X_{i}\right)}{h_{\theta_{0}}\left(t \mid X_{i}\right)} \mid Y=\mathbf{y}\right]
$$

hence the Monte Carlo score vector (4.11) is

$$
\nabla l_{k}(\theta)=\frac{1}{k} \sum_{j=1}^{k}\left\{w_{j, \theta_{0}, \theta} \sum_{\left(X_{i}, Z_{i}\right) \in W_{j}}\left[-\nabla H_{\theta}\left(X_{i}\right)+\sum_{t \in X_{i}+Z_{i}} \nabla \log h_{\theta}\left(t \mid X_{i}\right)\right]\right\}
$$

where as before $W_{j}$ is a sample of size $k$ from the conditional distribution of the complete data given $\mathbf{y}$ under the reference parameter $\theta_{0}$, and $w_{j, \theta_{0}, \theta}$ are the importance weights.

4.4.1 Example: Cox-Matérn cluster process Consider the Cox-Matérn cluster process on $\mathbb{R}^{2}$ with offspring governed by (3.2) and independent Poisson background clutter. Treating the range $R_{h}$ as fixed, the parameter vector is $\theta=(\epsilon, \mu)$. The grain is a finite point process on $\tilde{\mathcal{X}}=B\left(0, R_{h}\right)$, and $H(\xi)=\mu \pi R_{h}^{2}$ for each genuine parent $\xi \in \mathcal{X}$. For the dummy parent, $H\left(x_{0}\right)=\epsilon\left|\mathcal{X} \oplus B\left(0, R_{h}\right)\right|$. If $\mathcal{X}$ is a convex set, the Steiner formula may be used to find an explicit expression of this area, see section 2.3. By differentiation with respect to the parameter vector, it follows that the components of $\nabla l_{k}(\theta)$ are the weighted averages of $-\left|\mathcal{X} \oplus B\left(0, R_{h}\right)\right|+n\left(Z_{0}^{j}\right) / \epsilon$ and $-n\left(W_{j}\right) \pi R_{h}^{2}+\sum_{i=1}^{n\left(W_{j}\right)} n\left(Z_{i}^{j}\right) / \mu$ where $n\left(W_{j}\right)$ denotes the number of genuine parents in $W_{j}$, and $Z_{0}^{j}$ its orphan cluster. The EM-updates are easily derived:

$$
\begin{aligned}
\epsilon^{(n+1)} & =\frac{E_{\theta^{(n)}}\left[n\left(Z_{0}\right) \mid Y=\mathbf{y}\right]}{\left|\mathcal{X} \oplus B\left(0, R_{h}\right)\right|} \\
\mu^{(n+1)} & =\frac{E_{\theta^{(n)}}\left[\sum_{i=1}^{n(X)} n\left(Z_{i}\right) \mid Y=\mathbf{y}\right]}{E_{\theta^{(n)}}\left[\pi R_{h}^{2} n(X) \mid Y=\mathbf{y}\right]} .
\end{aligned}
$$




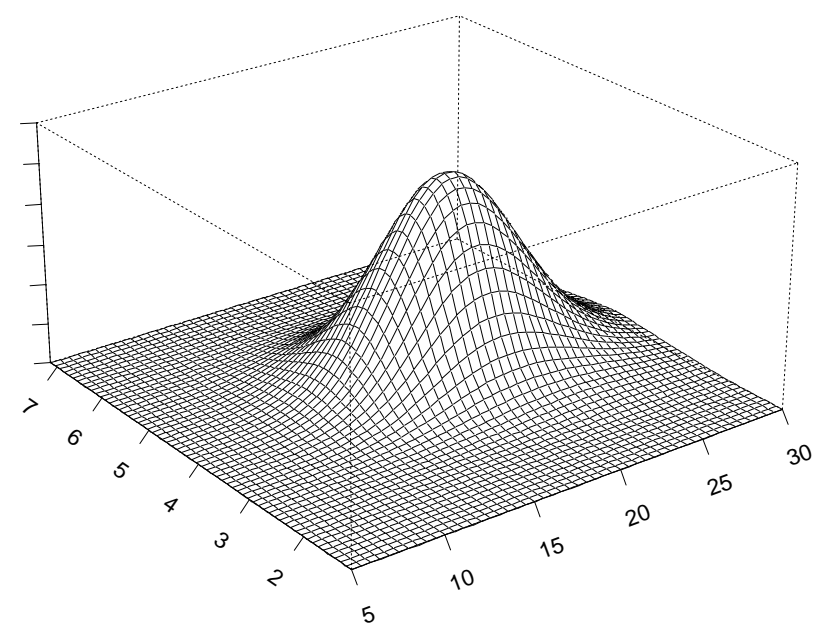

Figure 3: Monte Carlo log likelihood ratio surface as a function of the noise intensity $\epsilon \epsilon$ $(5,30)$ and the mean cluster size $\mu \pi R_{h}^{2} \in(1.14,7.14)$ for the redwood seedlings data (figure 1 (left)) based on a Cox-Matérn cluster process with $R_{h}=0.061$ and reference parameter values such that the average number of points per cluster is 2.14 , the noise intensity $\epsilon=10.0$. We used a hard core prior with $R=0.03$ and $\beta=1.0$. One hundred realisations were subsampled from a run of a spatial birth-and-death process over $2.0 \times 10^{4}$ time units after burn-in.

For the example on redwood seedlings (section 4.3.1) with a unit rate hard core prior at range 0.03 and reference parameter vector $(10.0,183.06)$ as in figure 1 (right), the Monte Carlo $\log$ likelihood ratio for $\epsilon \in(5,30)$ and $\mu \pi R_{h}^{2} \in(1.14,7.14)$ is given in figure 3 ; the solution of the Monte Carlo score equations is $\left(\hat{\epsilon}_{100}, \hat{\mu}_{100}\right)=(19.65,354.15)$. For comparison, the Monte Carlo EM-updates would be $\epsilon=15.12$ and $\mu=311.61$ corresponding to 3.64 daughters on average in a cluster.

\subsection{Adaptive coupling from the past}

Remarkably, the spatial birth and death approach described in section 4.3 can be adapted to yield an exact sample from the desired posterior distribution using coupling from the past 
$[70,46]$. Such algorithms are particularly efficient when there is some monotonicity in the state space, and the sampler respects this order.

In the context of this paper, the prior distribution of $X$ is a repulsive Markov point process. Whether the same is true for the posterior distribution depends on the grain distributions $Q_{\xi}$. However, for Cox cluster processes, we showed in section 4.2 that the posterior distribution is repulsive and hereditary too. Moreover, (4.8)-(4.9) reverse the inclusion ordering in the sense that if $\mathbf{x} \subseteq \mathbf{x}^{\prime}$ then $b(\mathbf{x}, \xi) \geq b\left(\mathbf{x}^{\prime}, \xi\right)$ for all $\xi \in \mathcal{X}$, while $d\left(\mathbf{x}, x_{i}\right) \leq d\left(\mathbf{x}^{\prime}, x_{i}\right)$ for $x_{i} \in \mathbf{x}$. Our proof can be found in [53, Section 4.9.3]. If the displacement functions $h(\cdot \mid \cdot)$ are uniformly bounded by $H$, the posterior inherits local stability from the prior. Such properties are particularly pleasing for Bayesian analysis, as they imply that the choice of prior is not crucial in these respects. Hence the coupling from the past algorithm of Kendall and Møller for locally stable point processes in principle applies [46]. Those authors presented their method for the constant death rate dynamics, with a dominating process that is Poisson with an upper bound to the conditional intensity of the distribution to be sampled as intensity. In our context, such a method would be impractical, as in most cases the upper bound will be orders of magnitude too large. For this reason, we present an adaptive coupling from the past algorithm based on (4.8)-(4.9).

Suppose a spatial birth-and-death process with transition rates $b(\cdot, \cdot)$ and $d(\cdot, \cdot)$ is available to sample from the posterior density of cluster centres $p_{X \mid Y}(\cdot \mid \mathbf{y})$, and we have upper and lower bounds

$$
\begin{aligned}
b(\mathbf{x}, \xi) & \leq \bar{b}(\xi) \\
d(\mathbf{x} \cup\{\xi\}, \xi) & \geq \underline{d}(\mathbf{x} \cup\{\xi\}, \xi)
\end{aligned}
$$

holding for all configurations $\mathbf{x}$ and all $\xi \in \mathcal{X}$. Suppose furthermore a unique probability density $\pi(\cdot)$ solving the detailed balance equations

$$
\pi(\mathbf{x}) \bar{b}(\xi)=\pi(\mathbf{x} \cup\{\xi\}) \underline{d}(\mathbf{x} \cup\{\xi\}, \xi)
$$

exists. For the classical constant death rate process, $\underline{d}(\mathbf{x} \cup\{\xi\}, \xi) \equiv 1, \bar{b}(\xi)$ is an upper bound to the posterior conditional intensity at $\xi$ that does not depend on the configuration to which $\xi$ is added, and $\pi(\cdot)$ defines an inhomogeneous Poisson process with intensity function $\bar{b}(\cdot)$. The generic adaptive choice in our context is

$$
\bar{b}(\xi)=\lambda \exp \left[-\int_{A} h(a \mid \xi) d a\right] \prod_{j=1}^{m}\left[1+\frac{h\left(y_{j} \mid \xi\right)}{\epsilon}\right] \leq \lambda \prod_{j=1}^{m}\left[1+\frac{h\left(y_{j} \mid \xi\right)}{\epsilon}\right]
$$

where $\lambda$ is the prior local stability bound. If a uniform bound is required McKeague chapter? the right hand side above may be replaced by $\lambda \sup _{\xi \in \mathcal{X}} \prod_{j=1}^{m}\left[1+\frac{h\left(y_{j} \mid \xi\right)}{\epsilon}\right]$. Similarly, for the transition rates given by (4.8)-(4.9), generic bounds are

$$
\bar{b}(\xi)=\lambda\left[1+\sum_{j=1}^{m} \frac{h\left(y_{j} \mid \xi\right)}{\epsilon}\right]
$$


and

$$
\underline{d}(\mathbf{x} \cup\{\xi\}, \xi)=\exp \left[\int_{A} h(a \mid \xi) d a\right]\left[1+\sum_{j=1}^{m} \frac{h\left(y_{j} \mid \xi\right)}{\epsilon}\right] / \prod_{j=1}^{m}\left[1+\frac{h\left(y_{j} \mid \xi\right)}{\epsilon}\right] .
$$

The corresponding equilibrium distribution is that of a Poisson process with intensity function $\lambda e^{-\int_{A} h(a \mid \xi) d a} \prod_{j=1}^{m}\left[1+\frac{h\left(y_{j} \mid \xi\right)}{\epsilon}\right]$. However, one may often do better by exploiting specific model characteristics, as we shall illustrate in section 4.6 below.

If we couple the spatial birth-and-death process defined by $\bar{b}(\cdot)$ and $\underline{d}(\cdot, \cdot)$ to processes defined by $b(\cdot, \cdot)$ and $d(\cdot, \cdot)$ as in [46], we obtain the following algorithm.

Algorithm 1 Let $V_{t, \xi}, t \leq 0, \xi \in \mathcal{X}$, be a family of independent, uniformly distributed random variables on $(0,1)$. Initialise $T=1$, and let $D(0)$ be a sample from $\pi(\cdot)$. Repeat

- extend $D(\cdot)$ backwards until time $-T$ by means of a spatial birth-and-death process with birth rate $\bar{b}(\cdot)$ and death rate $\underline{d}(\cdot, \cdot)$;

- generate a lower process $L_{-T}(\cdot)$ and an upper process $U_{-T}(\cdot)$ on $[-T, 0]$ as follows:

- initialise $L_{-T}(-T)=\emptyset, U_{-T}(-T)=D(-T)$;

- to each forward transition time $t \in(-T, 0]$ of $D(\cdot)$ correspond updates of the upper and lower processes;

- in case of a death (i.e. a backwards birth), say $D(t)=D(t-) \backslash\{d\}$ where $D(t-)$ denotes the state just prior to time $t$, the point $d$ is deleted from $L_{-T}(t-)$ and $U_{-T}(t-)$ as well;

- in case of a birth, say $D(t)=D(t-) \cup\{\xi\}$, the point $\xi$ is added to $U_{-T}(t-)$ only if

$$
V_{t, \xi} \leq \max \left\{\frac{b(\mathbf{x}, \xi) \underline{d}(\mathbf{x} \cup\{\xi\}, \xi)}{\bar{b}(\xi) d(\mathbf{x} \cup\{\xi\}, \xi)}: L_{-T}(t-) \subseteq \mathbf{x} \subseteq U_{-T}(t-)\right\}
$$

similarly, $\xi$ is added to $L_{-T}(t-)$ only if $V_{t, \xi}$ does not exceed the above expression with a minimum instead of a maximum;

- if $U_{-T}(0)=L_{-T}(0)$, return the common value $U_{-T}(0)$; otherwise set $T:=2 T$;

until the upper and lower processes have coalesced.

The next theorem gives conditions for algorithm 1 to output unbiased samples from the posterior distribution of cluster centres.

Theorem 1 Let $p_{X}(\cdot)$ be an anti-monotone, locally stable Markov point process density with respect to a unit rate Poisson process on a compact set $\mathcal{X} \subseteq \mathbb{R}^{d}$, and $h(\cdot \mid \cdot)$ a uniformly bounded displacement fuction of a Cox cluster process $U$ observed in a bounded window $A$. Suppose the birth rates $b(\cdot, \cdot)$ and death rates $d(\cdot, \cdot)$ define a unique spatial birth-and-death process converging in distribution to the posterior density of cluster centres $p_{X \mid Y}(\cdot \mid \mathbf{y})$, and 
there exist upper and lower bounds (4.12)-(4.13) also defining a unique spatial birth-anddeath process that converges in distribution to a probability density $\pi(\cdot)$ for which $\pi(\emptyset)>0$ and detailed balance between births and deaths holds.

Then the coupling from the past algorithm 1 almost surely terminates and outputs an unbiased sample from $p_{X \mid Y}(\cdot \mid \mathbf{y})$.

The proof is an adaptation to the inhomogeneous case of the proof in [46].

Proof : First, note that by assumption the dominating process $D(\cdot)$ is in equilibrium, its distribution being defined by $\pi(\cdot)$. Clearly, for all $T>0$,

$$
\emptyset=L_{-T}(-T) \subseteq U_{-T}(-T)=D(-T)
$$

and by construction the updates respect the inclusion order. Hence $L_{-T}(t) \subseteq U_{-T}(t)$ for all $t \in[-T, 0]$. Moreover, the processes funnel, i.e.

$$
L_{-T}(t) \subseteq L_{-S}(t) \subseteq U_{-S}(t) \subseteq U_{-T}(t)
$$

whenever $-S \leq-T \leq t \leq 0$. The first inclusion can be verified by noting that $L_{-T}(-T)=$ $\emptyset \subseteq L_{-S}(-T)$ and recalling that the transitions respect the inclusion order. Since $U_{-T}(-T)=$ $D(-T) \supseteq U_{-S}(-T)$, the last inclusion in (4.14) follows by the same argument. If $L_{-T}\left(t_{0}\right)=$ $U_{-T}\left(t_{0}\right)$ for some $t_{0} \in[-T, 0]$, as the processes are coupled, $L_{-T}(t)=U_{-T}(t)$ for all $t \in\left[t_{0}, 0\right]$.

Next, set $X_{-T}(-T)=\emptyset$ and define a process $X_{-T}(\cdot)$ on $[-T, 0]$ in analogy to the upper and lower processes, except that if $X_{-T}(t-)=\mathbf{x}$ the birth at time $t$ of a point $\xi$ is accepted if $V_{t, \xi} \leq \frac{b(\mathbf{x}, \xi) \underline{d}(\mathbf{x} \cup\{\xi\}, \xi)}{\bar{b}(\xi) d(\mathbf{x} \cup\{\xi\}, \xi)}$. In other words, $X_{-T}(\cdot)$ exhibits the dynamics of a spatial birthand-death process with birth rate $\tilde{b}(\mathbf{x}, \xi)=b(\mathbf{x}, \xi) \frac{d(\mathbf{x} \cup\{\xi\}, \xi)}{d(\mathbf{x} \cup\{\xi\}, \xi)}$ and death rate $\tilde{d}(\mathbf{x} \cup\{\xi\}, \xi)=$ $\underline{d}(\mathbf{x} \cup\{\xi\}, \xi)$. Thus, its detailed balance equations coincide with those for $b(\cdot, \cdot)$ and $d(\cdot, \cdot)$. Furthermore, $\tilde{b}(\cdot, \cdot) \leq b(\cdot, \cdot)$, hence explosion is prevented so that the process converges in distribution to its equilibrium distribution defined by $p_{X \mid Y}(\cdot \mid \mathbf{y})$. The inclusion properties derived above imply $L_{-T}(0) \subseteq X_{-T}(0) \subseteq U_{-T}(0)$, so that - provided the sampler terminates almost surely - with probability 1 the $\operatorname{limit} \lim _{T \rightarrow \infty} X_{-T}(0)$ is well-defined. Since $D(\cdot)$ is in equilibrium, $X_{-T}(0)$ has the same distribution as if the $X$-process were run forward from time 0 (coupled to the dominating process as before) over a time period of length $T$, the limit distribution of which is $p_{X \mid Y}(\cdot \mid \mathbf{y})$. We conclude that the algorithm outputs an unbiased sample from the posterior distribution of parents.

It remains to show that coalescence occurs almost surely. Recall that by assumption $\pi(\emptyset)>0$. Set, for $n \in \mathbb{N}_{0}, E_{n}=\mathbf{1}\{D(-n) \neq \emptyset\}$. Now $\left(E_{n}\right)_{n}$ is an irreducible aperiodic Markov chain on $\{0,1\}$ for which the equilibrium probability $\pi(\emptyset)$ of state 0 is strictly positive. Hence state 0 will be reached with probability 1 , which implies that the dominating process $D(t)_{t \leq 0}$ will almost surely be empty for some $t$. But then (4.14) and the coupling imply that the algorithm terminates almost surely, and the proof is complete.

\subsection{Example: Cox-Matérn cluster process}

To describe a tailor-made coupling from the past algorithm, consider a Cox cluster process with intensity function given by (3.1)-(3.2) and prior density (4.5). For this model, the birth 
rate (4.8) satisfies

$$
b(\mathbf{x}, \xi) \leq \beta\left[1+\sum_{j=1}^{m} \frac{h\left(y_{j} \mid \xi\right)}{\epsilon}\right]=\bar{b}(\xi)
$$

In order to derive a lower bound for the death rate (4.9), note that

$$
1+\frac{h\left(y_{j} \mid \xi\right)}{\lambda\left(y_{j} \mid \mathbf{x}\right)} \leq 1+\frac{\mu}{\epsilon+\mu} \leq 2
$$

if $y_{j} \in B\left(\xi, R_{h}\right) \cap U_{\mathbf{x}}$, where $U_{\mathbf{x}}=\cup_{x_{i} \in \mathbf{x}} B\left(x_{i}, R_{h}\right)$ denotes the union of balls centred at the points of $\mathbf{x}$. It follows that the death rate $d(\mathbf{x} \cup\{\xi\}, \xi)$ is bounded below by

$$
\underline{d}(\mathbf{x} \cup\{\xi\}, \xi)=d(\mathbf{x} \cup\{\xi\}, \xi) \prod_{j: y_{j} \in B\left(\xi, R_{h}\right) \cap U_{\mathbf{x}}}\left(\frac{1}{2}+\frac{\mu}{2 \lambda\left(y_{j} \mid \mathbf{x}\right)}\right) .
$$

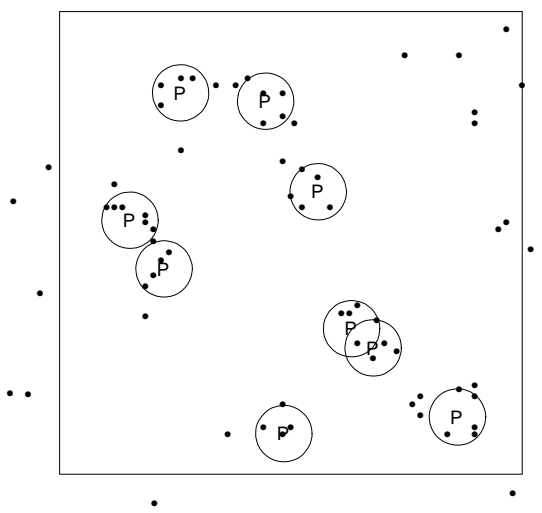

Figure 4: Realisation of extrapolated redwood seedling pattern on $\mathcal{X} \oplus B\left(0, R_{h}\right)$ from observations in unit square (box) and interpolated parent pattern (' $\mathrm{P}$ ') based on a Cox-Matérn cluster process with $R_{h}=0.061$ and $\left(\hat{\epsilon}_{100}, \hat{\mu}_{100}\right)$ obtained by coupling from the past. We used a hard core prior with $R=0.03$ and $\beta=1.0$. 
By the Preston theorem, the transition rates $\bar{b}(\xi)$ and $\underline{d}(\mathbf{x} \cup\{\xi\}, \xi)$ define a unique spatial birth-and-death process, whose limit distribution is given by

$$
\pi(\mathbf{x}) \propto \gamma^{-n\left(\mathbf{y} \cap U_{\mathbf{x}}\right)} \prod_{i=1}^{n(\mathbf{x})} \beta\left(x_{i}\right)
$$

a generalised area-interaction process $[7,32,45,88]$ with intensity function

$$
\beta(\xi)=\beta \exp \left[-\int_{A} h(a \mid \xi) d a\right] 2^{n\left(\mathbf{y} \cap B\left(\xi, R_{h}\right)\right)}
$$

and interaction parameter $\gamma=\left(\frac{1}{2}+\frac{\mu}{2 \epsilon}\right)^{-1}$.

Regarding the implementation of algorithm 1, note that

$$
\frac{b(\mathbf{x}, \xi) \underline{d}(\mathbf{x} \cup\{\xi\}, \xi)}{\bar{b}(\xi) d(\mathbf{x} \cup\{\xi\}, \xi)}=\mathbf{1}\{d(\xi, \mathbf{x})>R\} \prod_{j: y_{j} \in B\left(\xi, R_{h}\right) \cap U_{\mathbf{x}}}\left(\frac{1}{2}+\frac{\mu}{2 \lambda\left(y_{j} \mid \mathbf{x}\right)}\right)
$$

is decreasing in $\mathbf{x}$, so the sampler is anti-monotone, and the births in the upper and lower processes may be implemented by simply considering the current state of the other process at each transition, see [45].

We applied the above algorithm to the redwood seedlings data of figure 1 (left) for the Matérn parameter vector $(\epsilon, \mu)$ equal to its Monte Carlo maximum likelihood estimate (cf. section 4.4.1) and a hard core prior with $\beta=1.0$ and $R=0.03$ as before. A typical realisation from the posterior distribution of parents can be seen in figure 4 as well as an extrapolation of the redwood pattern to the set $\mathcal{X} \oplus B\left(0, R_{h}\right)$.

\section{ACKNOWLEDGEMENTS}

We gratefully acknowledge the expert programming support of Adri Steenbeek, very helpful comments from Yih Chong Chin, Nick Fisher, Richard Gill, Ilya Molchanov and Elke Thönnes, and the influence of previous unpublished collaboration with Andrew Lawson and Henry Y.W. Cheng.

\section{REFERENCES}

1. T. Arak, P. Clifford and D. Surgailis. Point-based polygonal models for random graphs. Advances in Applied Probability, 25:348-372, 1993.

2. T. Arak and D. Surgailis. Markov random fields with polygonal realizations. Probability Theory and Related Fields, 80:543-579, 1989.

3. T. Arak and D. Surgailis. Consistent polygonal fields. Probability Theory and Related Fields, 89:319-346, 1991.

4. A.J. Baddeley. Spatial sampling and censoring. In O. Barndorff-Nielsen, W.S. Kendall, and M.N.M. van Lieshout, editors, Stochastic geometry, likelihood, and computation. CRC Press/Chapman and Hall, Boca Raton, 1999.

5. A.J. Baddeley, N.I. Fisher and S.J. Davies. Statistical modelling and prediction of fault processes. In P.J. Hatherly, J. Shepherd, B.J. Evans and N.I. Fisher, editors, Integration of methods for the prediction of faulting, chapter 6, pages 143-176. Australian Coal Industry Research Laboratories, Sydney, March 1993. Final report on NERDDC Project 1588.

6. A.J. Baddeley and M.N.M. van Lieshout. Stochastic geometry models in high-level vision. In K.V. Mardia and G.K. Kanji, editors, Statistics and Images, Volume 1, Advances in Applied Statistics, a supplement to Journal of Applied Statistics, 20:231-256. Carfax, Abingdon, 1993. 
7. A.J. Baddeley and M.N.M. van Lieshout. Area-interaction point processes. Annals of the Institute of Statistical Mathematics, 46:601-619, 1995.

8. A.J. Baddeley, M.N.M. van Lieshout and J. Møller. Markov properties of cluster processes. Advances in Applied Probability (SGSA), 28:346-355, 1996.

9. A.J. Baddeley and J. Møller. Nearest-neighbour Markov point processes and random sets. International Statistical Review, 57:89-121, 1989.

10. R. Bendrath. Veralgemeinerung eines Satzes von R.K. Milne. Mathematische Nachrichten, 59:221-228, 1974.

11. J. Besag. Spatial interaction and the statistical analysis of lattice systems (with discussion). Journal of the Royal Statistical Society, Series B, 36:192-236, 1974.

12. D.A. Binder. Bayesian cluster analysis. Biometrika, 65:31-38, 1978.

13. P.G. Blackwell. Bayesian inference for a random tessellation process. Probability and Statistics Research Report 485/98 (revised July 1999), University of Sheffield, 1998.

14. C. Chatfield and A.J. Collins. Introduction to multivariate analysis. Chapman and Hall, London, 1980.

15. A.G. Chessa. Conditional simulation of spatial stochastic models for reservoir heterogeneity. $\mathrm{PhD}$ thesis, Technical University of Delft, Delft, The Netherlands, 1995.

16. J.P. Chilès. Modèlisation géostatistique de réseaux de fractures. In M. Armstrong, editor, Geostatistics, volume 1, pages 57-76. Kluwer, Dordrecht, 1989.

17. D. R. Cox and V. Isham. Point processes. Chapman and Hall, London, 1980.

18. N.A.C. Cressie. Statistics for spatial data. John Wiley and Sons, New York, 1991.

19. N.A.C. Cressie and A.B. Lawson. Hierarchical probability models and Bayesian analysis of minefield locations. Advances in Applied Probability, 32:315-330, 2000.

20. D.J. Daley and D. Vere-Jones. An introduction to the theory of point processes. Springer Verlag, New York, 1988.

21. A. Dasgupta and A.E. Raftery. Detecting features in spatial point processes with clutter via model-based clustering. Journal of the Americal Statistical Association, 93:294-302, 1998.

22. J.S. De Bonet. Multiresolution sampling procedure for analysis and synthesis of texture images. Computer Graphics Proceedings, pp. 361-368, 1997.

23. A.P. Dempster, N.M. Laird and D.B. Rubin. Maximum likelihood from incomplete data via the EM algorithm (with discussion). Journal of the Royal Statistical Society, Series B, 39:1-37, 1977.

24. J. Diebolt and C.P. Robert. Estimation of finite mixture distributions through Bayesian sampling. Journal of the Royal Statistical Societey, Series B, 56:363-375, 1994.

25. P.J. Diggle. On parameter estimation for spatial point processes. Journal of the Royal Statistical Society, Series B, 40:178-181, 1978.

26. P.J. Diggle. Statistical analysis of spatial point patterns. Academic Press, London, 1983.

27. P.J. Diggle, T. Fiksel, P. Grabarnik, Y. Ogata, D. Stoyan and M. Tanemura. On parameter estimation for pairwise interaction processes. International Statistical Review, 62:99-117, 1994.

28. B. Everitt. Cluster analysis. Heinemann Educational Books, London, 1974.

29. A.E. Gelfand and B.P. Carlin. Maximum likelihood estimation for constrained or missing data models. Canadian Journal of Statistics, 21:303-311, 1993.

30. C.J. Geyer. Likelihood inference for spatial point processes. In O. Barndorff-Nielsen, W.S. Kendall, and M.N.M. van Lieshout, editors, Stochastic geometry, likelihood, and computation. CRC Press/Chapman and Hall, Boca Raton, 1999.

31. C.J. Geyer and J. Møller. Simulation procedures and likelihood inference for spatial point processes. Scandinavian Journal of Statistics, 21:359-373, 1994.

32. O. Häggström, M.N.M. van Lieshout and J. Møller. Characterisation and simulation results including exact simulation for some spatial point processes. Bernoulli, 5:641-659, 1999. 
33. H.H. Haldorsen. Reservoir characterization procedures for numerical simulation. PhD thesis, University of Texas, Austin, 1983.

34. D.J. Hand. Discrimination and classification. Wiley, Chichester, 1981.

35. J.A. Hartigan. Clustering algorithms. Wiley, New York, 1975.

36. N.L. Hjort and H. Omre. Topics in spatial statistics. Scandinavian Journal of Statistics, 21:289-357, 1994.

37. I. Hodder and C. Orton. Spatial analysis in archeology. Cambridge University Press, 1976.

38. F. Huang and Y. Ogata. Comparison of two methods for calculating the partition functions of various spatial statistical models. Australian and New Zealand Journal of Statistics, 43:47-65, 2001.

39. R.A. Johnson and D. W. Wichern. Applied multivariate statistical analysis. Prentice Hall, New Jersey, 1982.

40. A.G. Journel. Geostatistics for conditional simulation of ore bodies. Economic Geology, 69:673-680, 1974.

41. A.G. Journel and C.J. Huijbregts. Mining geostatistics. Academic Press, 1978.

42. A.G. Journel and E.H. Isaaks. Conditional indicator simulation: application to a Saskatchewan uranium deposit. Mathematical Geology, 16:685-718, 1984.

43. L. Kaufman and P.J. Rousseeuw. Finding groups in data. An introduction to cluster analysis. Wiley, New York, 1990.

44. F.P. Kelly and B.D. Ripley. On Strauss's model for clustering. Biometrika, 63:357-360, 1976.

45. W.S. Kendall. Perfect simulation for the area-interaction point process. In L. Accardi and C.C. Heyde, editors, Probability towards the year 2000. World Scientific Press, 1997.

46. W.S. Kendall and J. Møller. Perfect simulation using dominating processes on ordered spaces, with application to locally stable point processes. Advances in Applied Probability, 32:844-865, 2000.

47. W.S. Kendall and E. Thönnes. Perfect simulation in stochastic geometry. Journal of Pattern Recognition, 32:1569-1586, 1999.

48. J.F.C. Kingman. Poisson processes. Oxford University Press, 1993.

49. C. Lantuéjoul. Conditional simulation of object-based models. In D. Jeulin, editor, Advances in theory and applications of random sets. World Scientific Publishing, 271-288, 1997.

50. G. Last. Some remarks on conditional distributions for point processes. Stochastic Processes and their Applications, 34:121-135, 1990.

51. A. Lawson. Discussion contribution. Journal of the Royal Statistical Society, Series B, 55:61-62, 1993.

52. M.N.M. van Lieshout. Stochastic geometry models in image analysis and spatial statistics. CWI tract 108 , Amsterdam, 1995.

53. M.N.M. van Lieshout. Markov point processes and their applications. Imperial College Press/World Scientific Publishing, London/Singapore, 2000.

54. M.N.M. van Lieshout and A.J. Baddeley. Markov chain Monte Carlo methods for clustering of image features. In Proceedings 5th IEE International Conference on Image Processing and its Applications, IEE Conference Publication, 410:241-245. IEE Press, London, 1995.

55. M.N.M. van Lieshout, I.S. Molchanov and S.A. Zuyev. Clustering methods based on variational analysis in the space of measures. Biometrika, 2001, to appear.

56. M.N.M. van Lieshout and R.S. Stoica. The Candy model revisited: Markov properties and inference. CWI Report PNA-R0115, August 2001.

57. M.N.M. van Lieshout and E.W. van Zwet. Exact sampling from conditional Boolean models with applications to maximum likelihood inference. Advances in Applied Probability (SGSA), 33:339-353, 2001.

58. J. Lund. Statistical inference and perfect simulation for point processes with noise. PhD thesis, The Royal Veterinary and Agricultural University, Copenhagen, 2000.

59. J. Lund, A. Penttinen and M. Rudemo. Bayesian analysis of spatial point patterns from noisy observations. Research Report, Department of Mathematics and Physics, The Royal Veterinary and Agricultural University, Copenhagen, 1999. 
60. J. Lund and E. Thönnes. Perfect simulation for point processes given noisy observations. Research Report 366, Department of Statistics, University of Warwick, 2000.

61. K.V. Mardia, J.T. Kent and J.M. Bibby. Multivariate analysis. Academic Press, London, 1979.

62. R.J. Marshall. A review of methods for the statistical analysis of spatial patterns of disease. Journal of the Royal Statistical Society, Series A, 154:421-441, 1991.

63. G. Matheron. Random sets and integral geometry. Wiley, New York, 1975.

64. B. Matérn. Spatial variation. Number 36 in Lecture Notes in Statistics. Springer Verlag, New York, 1986.

65. R.K. Milne. Identificability for random translations of Poisson processes. Zeitschrift für Wahrscheinlichkeitstheorie und verwandte Gebiete, 15:195-201, 1970.

66. J. Møller. On the rate of convergence of spatial birth-and-death processes. Annals of the Institute of Statistical Mathematics, 41:565-581, 1989.

67. A. Penttinen and D. Stoyan. Statistical analysis for a class of line segment processes. Scandinavian Journal of Statistics, 16:153-168, 1989.

68. C.J. Preston. Random fields. Springer Verlag, Berlin-Heidelberg-New York, 1976.

69. C.J. Preston. Spatial birth-and-death processes. Bulletin of the International Statistical Institute, 46:371391, 1977.

70. J.G. Propp and D.B. Wilson. Exact sampling with coupled Markov chains and applications to statistical mechanics. Random Structures and Algorithms, 9:223-252, 1996.

71. R.-D. Reiss. A course on point processes. Springer Verlag, Berlin-Heidelberg-New York, 1993.

72. B.D. Ripley. Modelling spatial patterns (with discussion). Journal of the Royal Statistical Society, Series B, 39:172-212, 1977.

73. B.D. Ripley. On tests of randomness for spatial point patterns. Journal of the Royal Statistical Society, Series B, 41:368-374, 1979.

74. B.D. Ripley. Spatial statistics. Wiley, New York, 1981.

75. B.D. Ripley. Statistical inference for spatial processes. Cambridge University Press, 1988.

76. B.D. Ripley. The use of spatial models as image priors. In A. Possolo, editor, Spatial statistics and imaging, number 20 in Lecture notes - monographs, pages 309-340. Institute of Mathematical Statistics, Hayward, California, 1991.

77. B.D. Ripley and F.P. Kelly. Markov point processes. Journal of the London Mathematical Society, 15:188192, 1977.

78. D. Ruelle. Statistical mechanics. Wiley, New York, 1969.

79. L. A. Santaló. Integral geometry and geometric probability. Encyclopedia of Mathematics and Its Applications, Volume 1, Addison-Wesley, 1976.

80. J. Serra. Image analysis and mathematical morphology. Academic Press, London, 1982.

81. M. Stein. Interpolation of spatial data: some theory for kriging. Springer Verlag, New York, 1999.

82. R. Stoica, X. Descombes and J. Zerubia. A Gibbs point process for road extraction in remotely sensed images. Research Report 3923, INRIA Sophia Antipolis, 2000.

83. R.S. Stoica, X. Descombes, M.N.M. van Lieshout and J. Zerubia. An application of marked point processes to the extraction of linear networks from images. In J. Mateu and F. Montes, editors, Spatial statistics: case studies, Advances in Ecological Sciences, Volume 13. WIT Press, Southampton, 2001.

84. D. Stoyan, W.S. Kendall, and J. Mecke. Stochastic geometry and its applications. Wiley, Chichester, 1987.

85. D.J. Strauss. A model for clustering. Biometrika, 63:467-475, 1975.

86. E. Thönnes. The conditional Boolean model revisited. Markov Processes and Related Fields, 7:77-96, 2001.

87. D.M. Titterington, A.F.M. Smith and U.E. Makov. Statistical analysis of finite mixture distributions. Wiley, Chichester, 1985.

88. B. Widom and J.S. Rowlinson. A new model for the study of liquid-vapor phase transitions. Journal of Chemical Physics, 52:1670-1684, 1970. 